\title{
INNOVATION AND IMPROVEMENT Interval Examination: Building Primary Care Teams in an Urban Academic Teaching Clinic
}

\author{
Reena Gupta, MD, Elizabeth Davis, MD, and Claire Horton, MD, MPH \\ Division of General Internal Medicine, San Francisco General Hospital, University of California, San Francisco, CA, USA.
}

KEY WORDS: team-based care; primary care redesign; patient-centered medical home; medical student and residency education.

$\mathrm{J}$ Gen Intern Med 28(11):1517-21

DOI: $10.1007 / \mathrm{s} 11606-013-2598-7$

(c) Society of General Internal Medicine 2013

\section{GMC AT BASELINE}

Until recently, residents, faculty, nurses, medical assistants and clerks in our urban academic clinic worked in silos. Far too often, part-time resident or faculty primary care providers (PCPs) were unavailable when their patients needed to be seen and patients saw different providers and staff each time they came to clinic. Our 118 staff and providers - who often did not know each other's names-had no structured systems for communication around ongoing patient needs. Confusion about roles and responsibilities led to duplication of work, lack of care coordination, and unnecessary delays in care. We tackled each day's set of crises as they developed, but the chaos never abated.

While the providers and staff at San Francisco General Hospital's (SFGH) General Medicine Clinic (GMC) had always been mission-driven people who advocated for patients, the clinic could be an overwhelming place to practice primary care. Providers often spent hours on nights and weekends calling patients, corresponding with pharmacies or medical equipment companies, completing disability forms, coordinating with home nurses or case managers, and following up on referrals and test results. The burden fell especially on residents. As one resident wrote, "I often feel like I'm on an island all alone. I am in clinic for over 4 hours after my clinic day ends and spend my free time in the evenings finishing notes, following up on referrals and calling patients.... It makes primary care feel like an incredible burden on top of busy, inpatient responsibilities."

Staff members were also frustrated, feeling they lacked relationships and had no way to communicate with many of the providers. Though the clinic achieved national benchmarks for clinical quality, our patients faced long wait

Received April 18, 2013

Revised July 11, 2013

Accepted August 2, 2013

Published online September 17, 2013 times, limited appointment and telephone access, low rates of continuity with PCPs and low patient satisfaction with access to care.

Collectively, we form the current medical leadership of the clinic; all of us were residents who trained in the GMC ourselves. We remember (and indeed still experience) the simultaneous inspiration and stress that the GMC creates. We needed to transform our clinic in order to address weaknesses in access, continuity, coordination, and resident experience - but how? We were not alone; the literature shows that academic clinics are often dysfunctional for patients and trainees alike, ${ }^{1-3}$ and that teaching clinics face significant challenges to medical home transformation. ${ }^{4-6}$ However, we found no clear roadmap in the literature for how to transform a large, complex teaching clinic like ours.

\section{GETTING TO TEAMS}

The clinic management team-our medical directors, nurse manager, practice manager, and senior clerk-started the transformation process in 2011 by completing a patientcentered medical home (PCMH) assessment. ${ }^{7,8}$ It identified three priority improvement areas: team-based care, enhanced access, and care coordination. We decided to focus on team-based care as a foundation upon which we would improve access, care coordination, and resident and staff experience. The impetus for transformation did not come from hospital mandates, residency program pressures, or financial incentives for PCMH recognition. Rather, the hospital leadership and our management team recognized transformation was essential to moving the clinic from a provider of last resort to a provider of choice in the era of health reform.

Many partnerships supported our transformation. We belong to the San Francisco Department of Public Health (SF DPH) primary care network, and each of us is deeply involved in shaping the system-wide PCMH transformation, funded through the Centers for Medicare \& Medicaid Services Incentive Program (CMSIP). With CMSIP funding, two of us received protected time as GMC assistant medical directors to lead our PCMH and complex care management implementation. We partnered with the University of California, San Francisco Center for Excel- 
lence in Primary Care (CEPC) and Coleman Associates for technical assistance and practice coaching. ${ }^{9,10}$ Finally, we identified local high-functioning medical homes and performed site visits. This helped our entire management team visualize the benefits of team-based care and identify practical steps to creating teams.

Resources needed to implement our team model included: 0.5 FTE for our two assistant medical directors, one registered nurse (RN) and one medical assistant (MA) for complex care management, three nurse practitioner (NP) full-time equivalents (FTE) for continuity, and external consulting from CEPC and Coleman Associates. Beyond this, we did not have funding to hire any additional staff or providers.

When we first proposed the idea of team-based care to providers and staff, we were met with enthusiasm as well as skepticism ranging from "Why do we need teams?" to "Aren't we all one big team already?" Some staff members who had participated in prior change efforts worried that this would be yet another project that would not stick. To address this reluctance and encourage culture change, we held meetings with all staff and provider groups to solicit champions from each discipline (RNs, MAs, clerks, behaviorists, NPs, residents, and attendings) to design our teams. With the clinic management group, these individuals formed our medical home committee. For 6 months prior to launching teams in 2012, the committee met every 2 weeks and made most system change decisions. For example, they decided to allocate staff to teams by balancing language skills and schedules, then drawing names at random in a public forum.

Some staff groups, such as MAs and clerks, preferred management to make decisions. Other staff groups, such as nurses, wanted to be more involved in decision-making. We adapted to each group, presenting a more complete structure in MA meetings while integrating nurses into each step - holding weekly meetings and planning site visits for them to see RN team roles at other medical homes. Not everyone embraced the changes initially, but more bought in as they saw improvements in patient care and clinic efficiency. Involving staff in the key decisions generated support and ownership in the process.

\section{TEAM STRUCTURE}

Our clinic staff includes 24 faculty, 52 residents, 7 NPs, 8 RNs, 13 MAs, 7 clerks, 3 behaviorists, 2 pharmacists, and 2 nutritionists. Faculty and residents practice one to two halfdays per week, and many staff also work part-time. Forming functional teams in such a large clinic seemed daunting at first.

After some trial and error, we reorganized staff and providers into three clinic teams (Fig. 1). We had three FTE each of RNs, NPs, and behaviorists, so we created three teams and divided other staff and providers equally among the teams. In addition to the RN, NP, and behaviorist, each team has 2.5 provider FTE (eight part-time faculty and 1618 residents), two clerical FTE, and three medical assistant FTE. A complex care management team, pharmacists, and nutritionists support all the teams. Each team has a panel of approximately 2,200 patients. This panel size is smaller than traditional primary care clinics because of higher patient complexity and smaller resident panel sizes.

Our staffing ratios of about three FTE support staff per provider FTE are slightly higher than national averages of 2.7 per physician FTE, but significantly lower than those of high-functioning medical homes which have four or more support staff FTE for each provider. ${ }^{1-13}$ We built teams with the staff we had, but our ratios continue to limit the scope of team members' roles. We are advocating actively for additional staffing resources.

To address issues of continuity for our patients and provide support for our part-time providers, we implemented several

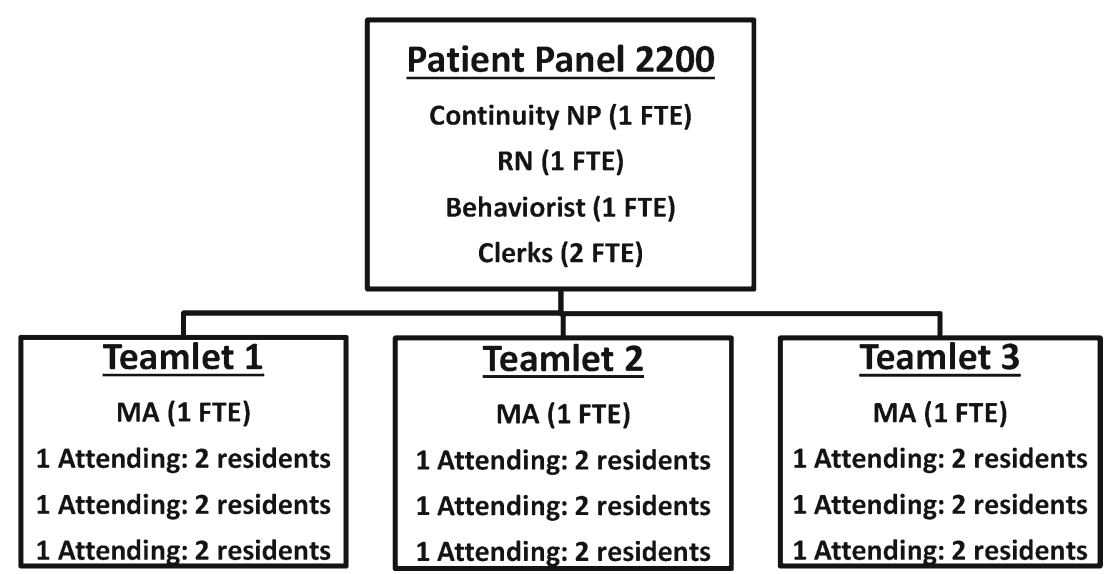

Figure 1. Organization of general medicine clinic care teams. 
partnerships within the team structure. In the past, patients frequently could not get an appointment with their PCP in a timely manner and saw whoever was available, resulting in low continuity rates. We integrated a NP continuity model, initially developed in 2007, into our clinic teams. If a resident or attending PCP is not available, the team NP sees the patient. Thus, patients can have same day or follow-up care with a provider who has long-term knowledge of their history and contact with the PCP. Continuity of care, defined as the percentage of visits in which a patient sees his or her own PCP or the team continuity NP, has increased from $43 \%$ before implementation of the new model to $89 \%$ afterwards.

Prior to launching the team model, providers worked with different MAs, often functioning independently. Communication between clinicians and MAs was sparse. In order to promote teamwork, this had to change. We made schedule adjustments aiming to pair each medical assistant with his or her teamlet providers (Fig. 1). When providers and MAs work consistently together, they become familiar with each other's styles, fostering strong communication and efficiency. Consistent pairing has not come easily. Provider schedules are highly variable, requiring our practice manager to spend several hours per week on this task. Feedback from staff and providers confirms the benefit for team functioning is well worth the investment.

Previously, when residents were away from clinic, they did not have coverage systems to follow-up on test results, referrals or patient messages. To provide such coverage, residents are paired with resident practice partners who are responsible for checking each other's inboxes. Though they sometimes need reminding and often desire more protected time to address inbox issues, residents express relief at having coverage and not being responsible for care coordination during busy inpatient rotations.

We have had primary preceptor assignments for several years; however, residents often precepted with any available attending and experienced limited continuity of clinical supervision. In order to strengthen resident-preceptor relationships, we developed new team preceptor guidelines and engaged faculty in their implementation. We schedule residents to be in clinic with their primary preceptor and encourage them to staff complex patients together to provide longitudinal clinical mentorship. The primary preceptor is also a resource for clinical questions or support outside of clinic. Preceptors have accepted spending more time supporting their residents outside of clinic, as they recognize the system has strengthened clinical supervision and continuity for residents and their patients.

Over the past year we have come to understand that realities of life impact our best laid plans. Prolonged medical leaves, budget cuts, and hiring freezes create short-staffing that disrupts scheduling and diminishes morale. Though part-time providers feel more supported with team partnerships, they still struggle to find sufficient time to coordinate care with their team. Having multiple team partners will never fully replace one full-time PCP who is in clinic every day, but we have found these systems of teamwork do improve care coordination.

\section{TEAM ROLE RE-DEFINITION}

After designing the team structure, we defined the roles of each member. The medical home committee met for 6 months to identify the clinic's tasks and match the right work to the right person. We eliminated duplicative or inefficient duties, creating space for team members to take on expanded roles.

For example, MAs now review patient records to identify healthcare maintenance gaps using the electronic health record (EHR). Though we had implemented standing orders for routine cancer screening, immunizations, and diabetic foot monofilament exams two years prior, they were not being completed consistently. With the MAs, we mapped out their healthcare maintenance workflows and found that hectic clinic flow was the major barrier. We reorganized MA schedules to provide each MA with 4 hours per week of dedicated chart preparation time and they are now consistently able to review patient records prior to clinic to identify healthcare maintenance gaps.

As another example, team clerks make appointment confirmation calls to patients, which has resulted in a $30 \%$ reduction in the clinic no-show rate. When we initially proposed confirmation calls, clerical staff expressed concern about being able to handle this new task. We eliminated less critical duties such as extraneous paperwork, creating space for them to take on this expanded role. After training the clerks with written telephone scripts, they are now more comfortable calling patients. Our patient advisory board members have declared our confirmation calls "the best thing that's happened to GMC in years."

GMC patients have a high burden of psychosocial as well as medical complexity, and coordinating their care often overwhelmed part-time providers. To address these challenges, we did two things. First, we integrated behavioral health onto our care teams. Each team has one behavioral health clinician - a licensed clinical social worker or psychologist - who is co-located in clinic. We reconfigured the roles of these existing staff to be team-based: they attend daily huddles to discuss behavioral health needs of scheduled patients; take warm hand-offs, meaning they meet with patients during provider visits, as well as schedule independent follow-up visits; and are available for consultation about behavioral health needs of team patients. Integrating behavioral health has allowed team members to communicate and address patients' psychosocial needs in a more coordinated manner. 
Second, we developed a complex care management team. Led by a registered nurse, this interdisciplinary team works intensively to decrease admissions and emergency department (ED) visits for our most frequently admitted patients. The team supports part-time providers by caring for patients between visits and focusing on time-intensive tasks such as self-management support. Patients in the program have $49 \%$ fewer inpatient hospital days (9.6 to 4.9 days/patient/ year) and $21 \%$ fewer ED visits (3.4 to 2.7 visits/patient/ year) compared to the year prior.

Despite these successes, redefining roles requires a continual juggling of responsibilities. One ongoing issue is the high volume of patients who drop-in to clinic every day, consuming RN time. While valuable, addressing drop-in patient needs limit $\mathrm{RN}$ availability for other team roles such as chronic care management. We have been working to improve our drop-in process as well as advocating for additional RN staffing to create capacity for expanded nurse team roles in the coming year.

\section{COMMUNICATION AND CHANGE}

Developing structure and defining roles is one thing. Actually making clinic teams functional is another. The key for us has been coaching and communication to create a culture of change in which staff and providers embrace new workflows and tasks. Over the past year, we focused on training teams to perform new roles, coaching to reinforce those roles, and developing specific protocols for communication.

After implementing teams we maneuvered resident and attending schedules to bring staff and providers together for three team training retreats, each lasting a half day. The agenda included: understanding team member roles, setting team ground rules, giving peer feedback, and team-building exercises. After overcoming some initial awkwardness, these retreats allowed the teams to form their identities. We continue to have quarterly retreats to reinforce team culture.

Recognizing that teams would require support and reinforcement, especially during the early weeks, we established a coaching program. The management group took on the bulk of the coaching, along with a few enthusiastic staff members. Coleman Associates consultants provided coach training. One coach was assigned to be on the floor every clinic session and provide one-on-one feedback and guidance to clinic staff and providers about their new team-based roles. For example, we coached MAs on closing the loop for healthcare maintenance with providers and offered feedback to improve communication during team huddles. After several weeks, we scaled back as staff and providers adopted the new processes.

Months later, however, we noticed some backsliding. People came late to huddles and confirmation call and chart preparation rates fell. Once again, we implemented focused coaching to reinforce teamwork. We have come to recognize that coaching is an ongoing process to support consistent performance. Our management team continues to meet weekly to review how teams are functioning and to identify further training needs.

We had previously piloted team huddles, but they never stuck because they were never a scheduled part of the day and team members were unclear about what to discuss. Recognizing that huddles are essential to team-based care, we addressed our prior barriers by (1) scheduling huddle times into clinic templates, (2) co-locating in team rooms for the huddles, (3) announcing the huddles overhead and by walking around the clinic, and (4) creating a structured huddle checklist and coaching teams to promote involvement of every team member. During huddles, teams review the day's patients, discussing the clerk's confirmation calls, MAs' chart preparation, behavioral health referrals, and anticipated needs of both nurses and clinicians (Fig. 2). While huddles initially took $15 \mathrm{~min}$, teams now review $15-20$ patients within $10 \mathrm{~min}$. Providers and medical assistants say that communicating about anticipated patient needs is the most valuable aspect of the huddles. The huddles also help reinforce roles. As an MA said, "I really like [the huddles]. It helps the residents see that we work with them, not for them."

Teams use several other forms of structured communication in addition to huddles. They meet monthly outside of clinic to review their team quality improvement data and discuss team processes. At these meetings, we have been gratified to see team members take ownership of the improvement process as they push their teams to improve huddles or brainstorm ideas for reducing no-shows. We also designed EHR workflows for team members to send messages to support team communication outside of clinic. Though we have implemented several communication systems, information still falls through the cracks. We understand that we will never have all providers in the same

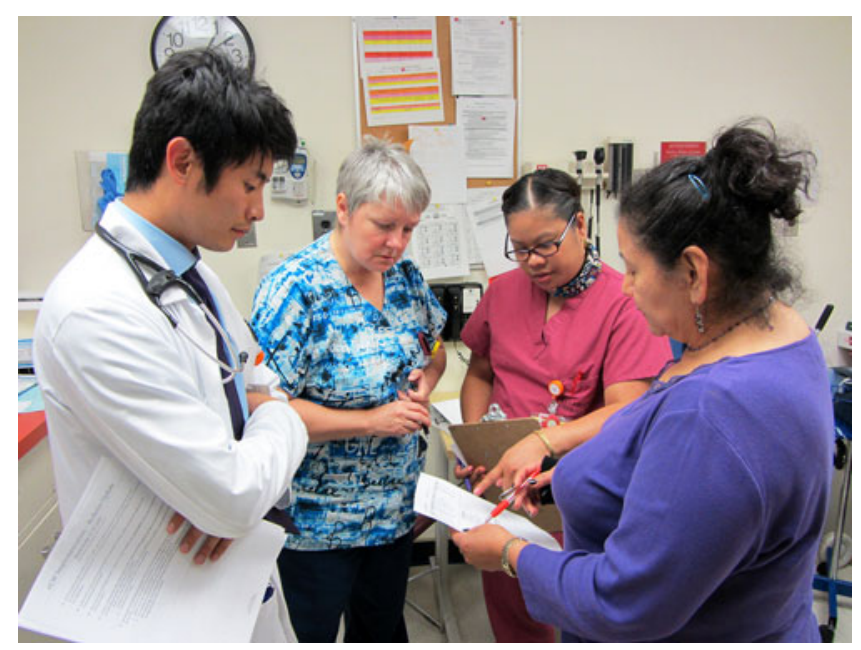

Figure 2. Multidisciplinary pre-clinic huddle. 
room at the same time, therefore we must remain committed to saying everything repeatedly in meetings, e-mails, memos and phone calls. And then probably saying it again.

We have had a clinic-wide data wall for several years - a prominent display of quality improvement data for staff and providers. It now includes weekly team metrics for each team member: clerical rates of confirmation calls, MA rates of chart preparation, and nurse visits per session. Though labor-intensive, the investment has proved worthwhile. Team members take pride in having the highest metrics and we have seen significant performance improvements. For example, in response to inconsistent healthcare maintenance completion rates of $40-60 \%$, we began tracking healthcare maintenance completion by team MAs. By providing and reviewing this data weekly, team MAs have now all reached greater than $80 \%$ completion.

Involving residents in practice transformation is fundamental to our culture of change. In addition to actively participating in team activities, residents lead quality improvement projects that have resulted in significant systems improvements, such as care management team communication, increasing vaccination and cancer screening rates, and reducing patients lost to follow-up. Residents continually help overcome hurdles, identify team solutions, and emerge with a strengthened appreciation for interdisciplinary relationships. However, as with all change, there have been unintended consequences. As confirmation calls improved show rates, residents feel the burden of a higher volume of patients. Also, after experiencing the benefits of MA pairing, staffing shortages that result in inconsistent scheduling frustrate residents. Some are also impatient with the seemingly slow pace of change, while others embrace practice transformation and believe the skills are essential to their training as physicians.

\section{THE ROAD AHEAD}

Over the past 2 years we have moved towards our goals: we have three teams that huddle daily; access and continuity have significantly improved; clinical quality scores continue to rise; and residents report improved clinic experience. As one resident said, "The largest impact that having teams at the GMC has had on me is this feeling that I'm not on my own advocating and caring for our patients - and that has been a huge emotional burden lifted."

We are by no means at the end of the journey. We must continue to expand team roles to provide more care coordination and further improve access so patients can be seen within their team promptly. With our teams in place, we feel poised to continue making these changes. Fundamentally, the successes and challenges of implementing our team model have rested on promoting a culture of change. We have learned a great deal from the wisdom and institutional memory of GMC staff members who have experienced many waves of change. And we have come to appreciate the importance of a sense of humor, humility, and a commitment to transparency in helping us traverse the long road to improvement.

Acknowledgments: We would like to thank the other members of our management team for their dedication: Brenda Barros, Jennifer Coffey, and Rosaly Ferrer. We are grateful to Kathryn Kruse for her diligent review of our manuscript. We also would like to acknowledge Dr. Thomas Bodenheimer of the UCSF Center for Excellence in Primary Care and Melissa Stratman of Coleman Associates for their contributions.

Financial Support: The authors declare that they have no funding sources to disclose.

Conflict of Interest: The authors declare that they do not have a conflict of interest.

Corresponding Author: Reena Gupta, MD; Division of General Internal Medicine, San Francisco General Hospital, University of California, 1001 Potrero Ave, SFGH Box 1364, San Francisco, CA 94110, USA (e-mail: Reena.gupta@ucsf.edu).

\section{REFERENCES}

1. Keirns CC, Bosk CL. The unintended consequences of training residents in dysfunctional outpatient settings. Acad Med. 2008;83(5):498-502.

2. Nadkarni M, Reddy S, Bates CK, Fosburgh B, Babbott S. Ambulatorybased education in internal medicine: current organization and implications for transformation. Results of a national survey of resident continuity clinic directors. J Gen Intern Med. 2010;26(1):16-20.

3. Weinberger SE, Smith LG, Collier VU. Redesigning training for internal medicine. Ann Intern Med. 2006;144(12):927-32.

4. Wagner EH. Academic, chronic care, and the future of primary care. J Gen Intern Med. 2010;25(S4):636-8.

5. Hern T, Talen M, Babiuch C, Durazo-Arvizu R. Patient care management teams: improving continuity, office efficiency, and teamwork in a residency clinic. J Grad Med Educ. 2009;1(1):67-72.

6. Markova T, Mateo M, Roth LM. Implementing teams in a patientcentered medical home residency practice: lessons learned. JABFM. 2012;25(2):224-31.

7. Patient-centered medical home assessment (PCMH-A). Available at: http://www.safetynetmedicalhome.org/sites/default/files / PCMHA.pdf. Accessed August 10, 2013.

8. Standards and guidelines for NCQA's patient-centered medical home (PCMH) 2011. National Committee for Quality Assurance. Available at: http://www.ncqa.org/PublicationsProducts/RecognitionProducts / PCMHPublications.aspx\#Standards. Accessed August 10, 2013.

9. Willard R, Bodenheimer T. The building blocks of high-performing primary care: lessons from the field. California Healthcare Foundation, April, 2012. Available at: http://www.chcf.org/ /media/ MEDIA\%20LIBRARY\%20Files / PDF / B / PDF\%20BuildingBlocks PrimaryCare.pdf. Accessed August 10, 2013.

10. Coleman Associates. Available at: http://www.patientvisitredesign.com/ coleman-associates. Accessed August 10, 2013.

11. Patel MS, Arron MJ, Sinsky TA, et al. Estimating the staffing infrastructure for a patient-centered medical home. Am J Manag Care. 2013;19(6):509-16.

12. Bodenheimer T. Building teams in primary care: lessons from 15 case studies. California Healthcare Foundation. July, 2007. Available at: http://www.chcf.org/ /media/MEDIA\%20LIBRARY\%20Files/PDF/B/ PDF\%20BuildingTeamsInPrimaryCareCaseStudies.pdf. Accessed August 10, 2013.

13. Klein S. The veteran's health administration: implementing patientcentered medical homes in the nation's largest delivery system. Commonwealth Fund; 2011. 